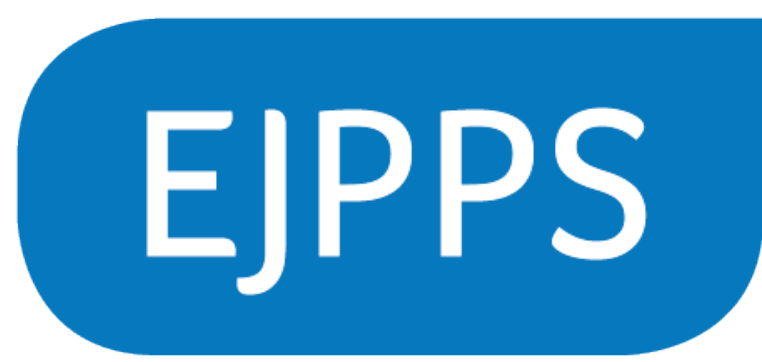

EUROPEAN JOURNAL OF
PARENTERAL AND
PHARMACEUTICAL SCIENCES

EJPPS - European Journal of Parenteral and Pharmaceutical Sciences Volume 25 Issue 4

https://www.ejpps.online/agar-desiccation-the-causes-and-how

https://doi.org/10.37521/ejpps25304

Solid Culture Media Desiccation

Agar Desiccation - The Causes and How to Address Them

Tammy Hassel Head of Microbiology - QC

Corresponding Author: Tammy Hassel, Microbiologist

PHSS Management Committee

England

Email: tamrynhassell@phss.co.uk 


\section{Agar Desiccation - The Causes and How to Address Them}

\section{Introduction}

Solid culture media, or agar plates, are prone to desiccation. The combination of warm, dry air and limited moisture content to achieve a solid finish leads to inevitable drying out and desiccation.

Cracks in the media, a loss of volume or shrinkage away from the sides of the Petri dish are evidence of desiccation. Overall, this reduces the capability of the media to support the growth of organisms. The reduction in growth support capability is particularly so if the microorganisms deposited on the surface of the media are stressed.

\section{Why is Desiccation an Issue}

It is a well-known fact that microbes have three basic requirements for growth; a food source, warmth and moisture. Reducing the moisture content risks inactivating and or inhibiting growth. The purpose of monitoring for microbes through the use of solid media is to capture microorganisms from the manufacturing environment and create favourable conditions to support their optimum growth, thus allowing them to replicate and form visible colonies for inspection.

Viable environmental monitoring is a challenging process to optimise for; you are looking for the chance of capturing one of the very few microorganisms present in your extensive manufacturing facility. To do so, you use solid culture media to take samples, snapshots in time, of your environment. Firstly, you have a vast space to monitor, and your monitoring activity needs to find the very few microorganisms present. If you have set your monitoring programme up suitably and you successfully capture organisms present in the environment but then add desiccation you risk losing critical data. Desiccation leads to a reduction in these favourable conditions and potentially a loss of critical data and false-negative results.

Desiccation of media is considered a significant quality issue as it can lead to the inhibition of growth and or cell death. The action of drying out of the media can lead to the formation of a skin on the surface. The skin formation inhibits the recovery of organisms, through "bounce" or "air bounce" (1) . As the purpose of environmental monitoring is to capture and support the growth of contaminants, this issue will result in inconclusive data gathering.

Desiccation is an inevitability. Cleanroom environments are by their very nature, high airflow facilities which, as you will see below, is a primary reason for the loss of moisture from agar plates. It is, therefore, important to ensure media qualification studies include a review that post use, your media, can meet the $70 \%$ growth rate recovery as specified in USP $<1227>$.

\section{How Does Desiccation Occur/Reasons Agar Desiccation Occurs}

A literature review and interview with suppliers was carried out to identify the causes of desiccation in solid media. It was noted, from this review, that desiccation is an unavoidable reality in the use of agar plates. A study carried out by Sandle T (2015), found it not unusual to experience weight loss, i.e. water loss - of between 10 to $20 \%$ of total weight, post-exposure to a cleanroom environment (2). It is noted that the study did not include reference to transit and pre-transit storage times.

The list below highlights the main reasons identified that lead to desiccation:

1. Aggressive airflow at exposure - cleanroom environments require high airflow rates at controlled temperature and humidity to ensure the flushing of contamination from the area. This combination of high airflow and a lower humidity increases the evaporative effect from the surface of the media, thus leading to desiccation. From one cleanroom environment to 
the next, you can see significant variability in environmental conditions. Therefore suppliers to the industry provide a range of solid media fills for manufacturing sites to select from, with $18 \mathrm{ml}, 25 \mathrm{ml}$, and $32 \mathrm{ml}$ fill plates being the most commonly available. It is however not unusual for sites to request custom fill plates to accommodate their unique needs.

It is worth noting that:

a. Turbulent airflow is less aggressive in the desiccation of plates.

b. Conversely, unidirectional airflow is known to lead to higher desiccation rates, and therefore any media qualification studies performed must consider the need to test the agar plates for suitability in these environments as a worst-case representation.

2. Hot and dry air - exposure to hot (warm) and dry air increase the rate of evaporation which leads to desiccation of media "the hairdryer effect". Cleanrooms and unidirectional airflow cabinets (UDAFs) naturally create "the hairdryer effect"; however, investigations can often overlook the incubators as a source of desiccation (see below). While manufacturers maintain cleanrooms at controlled temperatures to ensure operator comfort and drug stability, UDAFs have been known to produce significant heat loads. They, therefore, can be a source of hot and dry rapid airflow over the surface of solid media agar plates.

3. Aggressive fan units in incubators - as described above, incubators increase the hairdryer effect as their temperature settings tend to be higher than the ambient manufacturing and or laboratory environment. While agar plates are not exposed to the incubator environment directly, the very design of the petri dish is such that allows for ventilation of the air space to control for the formation of significant condensate on the lid. As such, when investigating desiccation sources unique to your site, it is suggested that plates be inspected for desiccation post-exposure and before incubation as well as post-incubation when reading takes place.

One way to monitor for significant moisture loss is to run humidity traces in your incubators for plates utilised in different environments. For example, plates used in media growth promotion studies, have very little exposure to the "hairdryer effect" as exposure is brief to inoculate their surfaces with the test organisms. Plates used in environmental monitoring have, in some cases, been exposed to the test environment for up to four hours or more. The moisture content of the agar from the same batch of media in these two use cases will be different prior to incubation. If you incubate these plates separately, you will see a difference in humidity in your incubators. The incubator fans pull more moisture out of the growth promotions plates (simply as there is more moisture still available within the agar) than from the environmental monitoring plates. As such, you will see higher humidity traces in the growth promotion plate incubator. It is necessary to control for this effect, as it could lead to condensation formation in your incubators which in turn can increase the risk of contamination.

4. Additives - neutralisers are known to contribute to the formation of cracks through the middle of the media as desiccation occurs. The addition of neutralisers forms chemical reactions within the media, reducing its strength, leading to sheering of the media and crack formation.

5. Storage time and conditions - extended storage of a plate, despite being wrapped in protective layers, at higher temperatures before use, allows increased time for evaporation to take place, especially if the wrapping utilised is optimised for gamma irradiation and not 
moisture control. While labels instruct that storage at $2-25^{\circ}, \mathrm{C}$ is acceptable. Where desiccation issues exist, ideally, ambient storage should not exceed $15^{\circ} \mathrm{C}$.

It is essential to understand the material qualities of the wrapping used to protect plates. As alluded to above, certain materials, such as those of nylon make up, are optimised for irradiation to increase the passage of dosage and have higher permeability properties, thus allowing moisture out of the packaging. I advise that during media qualification studies, you consider shelf-life storage. Observe the media for the formation of growth and or condensation within the packaging. Your observations may alert you to an issue with the packaging.

6. Broad temperature range changes - rapid and extreme temperature changes in storage and in-use can lead to excess water loss from media. For example, the moving of the plates between refrigerated storage and ambient cleanroom environments. While it is acceptable to utilise refrigerated storage media in your environmental monitoring programme with appropriate controls to bring the media to ambient conditions before use, give consideration to whether this may be leading to desiccation issues.

7. The concentration of agar in the plate - for solid media agar that requires irradiation to ensure sterility, suppliers can add additional agar to the media to better 'fix' the water into the medium. My findings show that irradiation can lead to water loss and that desiccation after that can be an unavoidable issue. Increased agar can lead to increased sheering forces when moisture is lost and thus may be the cause of crack formation in your media.

8. Consistency and variability of the supplier's manufacturing process - minor changes or variability in manufacturing processes of media can lead to changes in media performance at a site. These variations include a change of supplier for base agar formulations. If you observe a step-change in the number of desiccated plates, check with your manufacturer for modifications to the manufacturing process. Consider even like for like changes in your assessment of possible root causes for desiccation.

Another area for consideration is the transport of media to your site. Has the supplier experienced any issues in its logistics management of your supply of plates, are the plates stored for longer than previously seen, for example, is there a new carrier transporting your media to site.

9. Particle bounce - particles bouncing off the surface of media can increase the evaporation effects across the surface of an agar plate. Particle bounce is more likely to be an issue in higher particulate environments and as such is not considered a problem for low bioburden cleanroom facility or aseptic manufacturing site. This issue is noted here to inform the reader that it should be a consideration if your site is likely to experience this type of environmental challenge.

10. High doses of irradiation - high doses of irradiation can lead to chemical alteration (oxidation) of the media, which in turn leads to desiccation issues. A dose of $10 \mathrm{kGy}$ is considered safe for the sterilisation of media and suppliers across industry use between $10-$ 20 kGy. Doses above $15 \mathrm{kGy}$ are a potential source of investigation should you see desiccation in your solid media.

11. Failure to validate exposure times - not all media are created equal or will be suitable to use on your site. When purchasing new media, validate your exposure times to show that the 
media is capable of supporting growth post-exposure. Failure to do so may mask the fact that the media to be used on-site may not be suitable for the intended use and that your exposure times provide too much of a challenge to moisture management within your media and lead to desiccation issues.

\section{A Note on Incubators}

The section above has described several reasons for desiccation. It is, however, important to point out that incubator selection can play a significant role in desiccation issues. I have already noted that aggressive incubator fans can increase the evaporative effect from media.

During this review and from experience working in the industry, I have noted that the selection is not as simple as going out to tender for the size and temperature range you require. The control of the incubator fan unit (amongst other parameters) is a necessary consideration. There are incubators designed specifically for desiccation - you might see them used as dryers, but others are less than evident in this design intent and marketing material. Incubators that run at higher temperatures (above 30 degrees $\mathrm{C}$ ) and at $100 \%$ fan speed with no ability to control for this speed are very likely to lead to desiccation issues. When purchasing incubators, consider their use for your site and media specifically. Consider the control of the fan speed unit and discuss the fan unit's appropriateness for your needs with the supplier and your validation team at the User Requirement Specification drafting and Design Qualification stages.

\section{Solutions for Desiccation}

If you are facing issues of desiccation, below are several suggested remedies to be implemented to ensure no impact to the monitoring for microbiological control.

\section{Short Term Solutions}

As an immediate solution, assess whether any of your alternate approved suppliers, on-site supplies or different media types you have access to can be used. At the same time, you investigate the issue and implement the necessary changes required. Doubling up of a duplicate plate at your monitoring locations might be an option where practicality allows. Consider whether the doubling up of plates decreases efficiency or increases the risk of contamination from operators dropping items in media, not having sufficient space to carry out their tasks in controlled environments or the location of the media has nowhere to go due to the design of the monitoring point.

Alternatively, you could decrease the exposure time of the media (check the alignment of your current usage with your validation of the media) and change your media out more frequently per manufacturing session. Again consideration must be given to your volume of supply and the upstream impact on your QC lab due to increased volume and deliveries and the associated need to perform growth promotion and other media qualification activities. Also, your QC team will be handling many more plates and needing to record additional data per sampling location and manufacturing session - is your environmental monitoring documentation system set up to handle this change?

Media stored up to its expiry date is potentially at higher risk of desiccation dependant on the storage conditions used. In some circumstances, rejection of this media for use on-site and a shortening of the shelf life for the period of investigation can help reduce the number of desiccated plates. 


\section{Medium Term Solutions}

A more medium-term consideration is assessing the layout of your incubator shelves and seeing whether there is direct airflow from the fan units at the back/top and your plates showing desiccation. Revalidation of the layout would be required.

The use of parafilm or boxing up of your plates when in the incubator may also help reduce the level of desiccation seen. Both of these actions require revalidation of your media's capability to recover organisms under these new conditions.

A review of the fan speed settings on your incubators should be carried out by the engineering team to determine if this can be optimised to reduce speed but still ensure equal distribution of temperature (and humidity if appropriate) throughout the incubator.

\section{Longer Term Solutions}

An alternate media supply may be the only way to address the desiccation issues. Sometimes changes are not within the control of the manufacturing site. For example, changes to the supply, to packaging materials or at the media manufacturing site.

Approach your supplier and ask them to support your investigation. Often they may have different formulations or fill volumes they can offer that reduce the complexity of changing supply and thus the time during which you need to manage with the ongoing issue. You could ask for increased agar in your formulation. Consider the need for neutralisers in all settings (control of this can be tricky, but some suppliers provide media in differently coloured Petri dishes to help operators understand which media to use where) and investigate whether it is possible to lower the irradiation dose given to your plate supply.

The implementation of humidity changes can improve the conditions during incubation, but bring with them their own set of challenges and contamination risks. Any addition of moisture to the incubation environment is considered high risk for extrinsic contamination and the potential for false positives. A robust risk assessment and control strategy will be required to implement such changes.

Do not assume that the media may be solely at fault, consider changes to your local manufacturing environment where requalification or validation of a new facility may have lead to airflow and environmental condition changes.

\section{Solutions not considered}

Manufacturing sites will have different approaches to managing desiccated plate results. Some may choose to invalidate the result while others may be able to justify the reporting of the result under certain conditions. Throw in the added complication of a desiccated plate with growth on it, and you have a difficult decision to make.

In my experience, choosing to report results rather than assess a gap in your monitoring through invalidation of the result, is a significant challenge to justify and overcome. The carrying out of growth promotion testing is an argument put forward to qualify a result, but at what point do you decide that a plate is "too desiccated" to do further testing and how do you test for that precise condition of desiccation for all challenge organisms, how do you decide when a crack is too wide representing what percentage loss of surface? Even if you could average out the weight of your media pre and post use to give some indication of the level of water loss you were willing to accept, the variability in the weight of the plastic Petri dishes is such that it makes this argument too challenging to justify rationally. 
My suggested approach is to invalidate your result and assess the remaining environmental monitoring holistically to determine maintenance of the microbiological control of the environment. Where you observe growth on a desiccated plate, report the result and assume as a potential underreporting of a contamination event. Thus, assess the control measures in place, and further downstream or supporting in-process testing to assure the quality of the batch from a microbiological standpoint.

I am not saying that it is not possible to accept your desiccated plate results, but rather a word of caution on how this could be justified when challenged.

\section{Conclusion}

This review has identified various contributory factors that may be a cause for the desiccation of solid culture media. In summary, the inherent nature of the cleanroom environment and the associated need to expose media to aggressive air flows coupled with formulation sensitivities in the manufacturing of the media can result in a product not suitable for use at your site. However, with this knowledge, when assessing media for use on-site, better care can be taken in ensuring suitability for the unique environment you are managing.

\section{Notes}

1. It is important to note here that different organisms respond to this media stress differently and this should be considered should verification be carried out on media performance post desiccation. 


\section{References}

2. Sandle, T. (2015), Settle plate exposure under unidirectional airflow and the effect of weight loss upon microbial growth. European Journal of Parenteral and Pharmaceutical Sciences 2015: 20(2): 45-50 2015 PHSS 